\title{
Categorical Analysis on Mental Health Status of the people of Kerala during the COVID-19 Pandemic using a Proportional Odds Model
}

\author{
Krithi M.R. ${ }^{1}$, Shanimol Emerson ${ }^{2}$ \\ ${ }^{1} \mathrm{Ph} . \mathrm{D}$. Econometrics, Department of Econometrics, University of Madras, Chepauk, Chennai \\ ${ }^{2}$ M.A. Economics, University of Madras, Chepauk, Chennai. \\ Corresponding author: Krithi M.R. \\ Email - krithinair123@gmail.com
}

\begin{abstract}
Background: Covid-19 pandemic forced people to get confined to social isolation and maintain social distancing which affected their mental health. The study aims at understanding the impact on the mental health status of people in Kerala, based on their demographic profile and the psychological issues faced during the Covid-19 lockdown.

Methodology: The study included 700 respondents from various districts of Kerala telephonic and mailed questionnaires for the period of December 2020 - May 2021. Ordered logit model is used for the econometric analysis using $\mathrm{R}$ software.

Results: The overall mental health status of an individual is affected by various reasons which are taken as various categories for the analysis. As a result of log odds estimated, Covid-19 patients are more disposed to mental health issues. Male respondents had a positive impact on mental health issues and unmarried people of all gender are disturbed with lockdown distress. Almost all occupational categories taken into account are more or less shaken with the pandemic except those with proper job security including Government and PSU workforces. Psychological, physical well-being is affected by the pandemic lockdown overall mental health system of people of Kerala.

Conclusion: Covid-19 pandemic have affected the overall mental health of the people regardless of age and profession which can be more or less solved by the individuals themselves through yoga, meditation, exercise, reading books, gardening, cooking, virtual contacts with friends and family, getting enough sleep, etc. which helps tocan keep the mind relaxed and stable.
\end{abstract}

Keywords: Covid-19 lockdown, mental health, ordered logit, odds estimates, categorical analysis, Kerala, R software.

(Paper received $-8^{\text {th }}$ July 2021, Peer review completed $-5^{\text {th }}$ September 2021)

(Accepted $-18^{\text {th }}$ October 2021, Published $-5^{\text {th }}$ January 2022)

\section{INTRODUCTION}

The outbreak of the Covid-19 pandemic in China, by the end of 2019, forced millions of people to get confined to their homes and maintain social estrangement. These restrictions led to social isolation, which showed a sign of psychological distress and disorder that paved the way towards suicide attempts. An individual's emotion and personality are affected by both social and biological factors that enhance them to line in a tuneful environment. Mental health is a well-adjusted condition of characteristics and emotions. For productive and constructive work to be performed, one has to be in a work to be performed, one has to be in a state of proper mental well-being. Each demographic group faces different levels of mental soundness, on various occasions. Therefore, it is crucial to evaluate the emotional distress and social disorders among different groups of people based on their age, gender, marital status, etc. Covid-19 generated fear and anxiety among the public around the world. People with a special risk of stress manipulated a chance of depression 
and other mental health issues. According to Psychology Today, quarantined persons were found to be having a high prevalence of psychological distress, including symptoms of depression and Post Traumatic Stress Disorder (PTSO). The immense anxiety and worries generated due to Covid-19 are quite overwhelming. In addition to social distancing, the threat of losing the job, financial instability, sickness, loneliness, etc. affected mental health to a large extend.

Covid-19 turns to be a public health emergency and poses a challenge to emotional elasticity even in Kerala. From numerous daily Covid-19 cases to thousands of cases reported per day made both people and government enact more conscious measures to overcome the crisis. To slow down the rapid spread of the pandemic, lockdown measures have been put in place, the ability of people to leave their homes and interact with other people has been incomplete. The major public health concern relates to how these measures impact people's mental health. Interest in understanding how much the Covid-19 lockdown affected the mental health of people in Kerala since we understood the fact that India's public landscape has dramatically transformed over the last few months, with the most prolonged lockdown that the present generation can remember which was declared on 24th March 2020. Different studies from different parts of the world show that there is a high level of mental distress in various forms due to the lockdown imposed.

An online survey was carried out on socio-demographic variables, precautionary measures, awareness, and concerns regarding COVID-19 and mental health status during the lockdown. The contentment of details received was defensive against negative mental health, lesser number of checking for information on COVID-19, diminished self-perceived likelihood of constructing COVID-19, and trust in physician's capacity to recognize COVID-19 infection. This study identified the risk factors of mental health during the COVID-19 pandemic [1]. In the state of Maharashtra, a study was initiated with a total of 324 responses, which showed that, those who are less than 20 years were more depressed in the lockdown crisis than those who are above 20 years. When compared on the stress scale it was found that females of age less than 20 years were having more stress [2]. Another study aimed to assess the impact of the COVID-19 outbreak on mental health and the traumatic distress, a sample of 1,591 was analysed using Bivariate latent change score (BLCS) modelling to investigate the ability of pre-outbreak sense of coherence levels to predict changes in psychopathological symptoms. The finding is there was no change in psychopathological symptoms [3]. The impact of the unprecedented environment on the mental health of individuals and communities in its magnitude. They discussed some emerging concepts of the causal pathways of mental disorders and suggested strategies to prevent and promote mental health in individuals during this crisis [4]. Since quarantine can impact mental health, their study aimed to estimate the prevalence of depressive symptoms, anxiety symptoms, and sleeping disturbances in the Italian population during the lockdown with a sample size was 1515. Rising age, anon-existence of work-related troubles, and being married or being a cohabitant reduced such a probability. Being female, and increased time spent on the internet, and avoidance of actions through peer pressure amplified the probability of at least one mental health consequence. Females and participants with chronic illness were linked with the close-down of the economy with the predominance of sleep disturbances [5]. The real-time survey pieces of evidence from Germany, the US, and the UK showed that the COVID-19 impacts on the labour market differ across countries. Workers in Germany have a wellestablished short-time work scheme so substantially they are less likely to be affected by the pandemic. Within countries, the impacts are largely dissimilar and aggravate existing inequalities [6]. UK Household Longitudinal Study to find out how the pandemic is related to the situation of working parents who had to manage between the two life domains of work and home. They found that mental health in the United Kingdom is worse for working parents and that it is especially related to the increased financial insecurity and the time spent on childcare and home-schooling. They found that the burden is not shared equally between men and women, and between richer and poorer households [7].

\section{Need for the Study}

The Novel Coronavirus created massive distress in the psychological and emotional wellbeing of people. The rise in the number of Covid-19 cases in Kerala, generating fear and anxiety among various demographic groups. The government's Stay at Home policy to maintain social distancing showed a negative sign of social isolation, fear of contagion, chronic stress, and other disorders in the individuals, which again generated an impact on the increase in suicide rate in India and other countries. Psychologists infer that mild 
mood swings and disorder prone to suicidal behaviour among the public. Therefore this study plans to understand the soundness status of people, belonging to various demographic groups, and the possible suggestions to reduce their mental illness during the Covid-19 pandemic period.

\section{Aims and Objectives}

- To analyse the impact of Covid-19 lockdown on the mental health status of people in Kerala, based on their demographic profile.

- To check the influence of various mental health disorders on the overall mental health status of individuals.

- To understand the psychological issues faced during the lockdown and the possible solutions they adapt to overcome the same.

\section{METHODOLOGY}

The primary data has been collected through telephonic and mailed questionnaires for the period of December 2020 - April 2021 to understand the behavioural pattern of people throughout the lockdown period using R software. The choice of the starting period is constrained by the collection of data, which created certain limitations as the situation demands. 700 people from available districts of Kerala having various ages, gender, occupation, marital status, income, etc. responded to their mental health status during the Covid-19 pandemic and the possible mental health hacks they followed to overcome their issues.

\section{Measurement of Variables}

The following categorical variables are used for the analysis:

- Age: Age category has been divided into 5 dummies [11-17, 18-28, 29-38, 38-55, 56-77]

- Occupation: People belonging to various jobs are grouped into 7 categories [IT Professionals, Students, Housewives, Self-employed, Business, Daily wage workers, Governments, PSU Employees).

- Gender: Gender has 3 categories given as Male, Female, and Others.

- Marital Status: Marital status is categorized as Married, Unmarried, and divorced.

- Monthly Income: It holds 5 income dummies including [less than 10,000,10000-30000, 3000050000,50000-70000, above 70000]

- COVID-19 patients: The variable is used to check whether the respondent tested Covid positive at least once during the pandemic. Yes or No

- Threat to Well Being: This variable describes whether the pandemic showed a threat to the wellbeing of the respondent.

- Mental Health Status: It is necessary to check whether the respondents faced any issues with their mental health. Have issues / No issues.

- Risk Analysis: The variables demand if the respondents had faced any kind of risk on aspects of their health, job, finance, etc.

- Low/Medium/ High

- Overall Rating on Mental Health: This is the dependent variable used for the analysis that develops how the individual rate their mental health overall as [Excellent, Very Good, Good, Fair, Poor]

\section{Econometric Methodology}

\section{Ordered Logit}

Logit model is used in regression when a dependent variable is binary, and it takes 0 and 1 as dummy as the output predicted values. The probability of the dependent variable is estimated to be 1, i.e., $Y=1$.Ordinal response variable is analyzed using ordered logit model which is based on the cumulative probabilities of the response variable. The logit of each cumulative probability is assumed to be a linear function of the covariates with regression coefficients constant across response variable categories. Ordered logit is used 
when dependent variable has more than two categories and the values of each category have a sequential order in a meaningful manner and the value is higher than the previous value. When the response variable of interest taken into consideration is ordinal with more than 3 categories, it is advisable to use specific models such as the ordered logit model. Suppose if the variable (dependent variable) with $k$ categories where $X i$ is the vector of covariates. Having a normal regression model that shows a relationship between the covariates and probabilities of $k$ categories, whereas in an ordered logit model, the responses are analysed based on the cumulative probabilities and not in terms of probabilities of the categories

An ordered logit model for an ordinal response $Y i$ with $k$ categories is defined with the help of $C=k-1$ equations where the cumulative probabilities $g C_{i}=\operatorname{Pr}\left(Y_{i} \leq Y_{k} \mid X_{i}\right)$ are related to a linear predictor $\beta^{\prime} X_{i}=\mid \beta_{0}+\beta_{1} X 1_{i}+\beta_{2} X 2_{i}+\ldots$ through the logit function: $\beta$ Logit $\left(g k_{i}\right)=\log \left(g k_{i} /\left(1-g k_{i}\right)\right)=\alpha k-\beta^{\prime} X_{i}$, where $k=1,2, k-1$.

The parameters like $\alpha k$, are called thresholds or cut points, are in increasing order $(\alpha 1<\alpha 2<\ldots<\alpha \mathrm{k}-1)$ that determines the order of the dependent variable. Slopes $\beta$ having a vector is not indexed by the category index $k$, therefore for every response categories (dependent variable) the effects of the covariates are constant, known as the parallel regression assumption. While plotting Logit $\left(g k_{i}\right)$ against a covariate produces $k-1$ parallel lines, the minus before $\beta$ shows that there is an increasing covariate with a positive slope in association with a shift towards the right-end of the response scale, namely with respect to a rise of the probabilities of the higher categories. As the parallel regression assumption implies the proportionality of the odds of not exceeding the $k$-th category odds $k_{i}=g k_{i} /\left(1-g k_{i}\right)$, ordered logit model is also known as the proportional odds model.

\section{RESULTS}

The analysis sticks around 700 respondents using the categorical variables - age, gender, monthly income, occupation, marital status, covid status, risk analysis, mental health rating, etc.

The ordered logit model has been taken into account for understanding the probability of the available responses and for prediction. The analysis has been done using $\mathrm{R}$ software. Considering the interpretation of each variable:

Dependent Variable: Overall rating of mental health is the response variable in the ordered logistic regression. Intercepts are the predictors in the models and the cut points for the adjacent levels of the latent response variable. Those who receive a latent score(intercept) less than -2.79 are classified as "Poor", those who receive a latent score between -0.80 and -2.79 are classified as "Fair", those who receive a latent score between -0.80 and 1.05 are classified as "Good", between 1.05 and 2.37 are "Very Good", and those greater than 2.37 are classified as "Excellent".

\section{Coefficients:}

Age: This is the ordered log-odds estimate of comparing 18-28, 29-36, 39-55, and 56-76, to 11-17 keeping 11-17 as the base category, on expected Overall health status given the other variables are held constant in the model. The category of $18-28$ affects more than the reference category on mental health status, whereas 29-36,39-55,56-76 when compared to the 11-17 category, shows a negative effect on mental health status.

Gender: The effect of male ordered log-odds estimate when compared to the female category, 0.284 which affects more than the reference category on mental health status, keeping all the other variables held constant. Whereas other gender categories have a negative effect related to reference category on overall mental health status.

Marital Status: The ordered log-odds keeps divorced as the base category, on expected Overall health status The effect of the married ordered log-odds compared to the divorced category affects less than the reference category on the dependent variable whereas unmarried has a positive effect on Overall mental health status than others.

Monthly Income: All the income categories taken into account, when compared to the 10000-30000 category shows a negative effect on mental health status. Here we compare 30000-50000, 50000-70000, 
above 70000 and above 70000 , to $10000-30000$ with 10000-30000 as the base category, on expected Overall health status given the other variables are held constant in the model.

\section{Occupation:}

Daily wage workers, Government/PSU Employees, Housewives/Self Employed, IT Professionals, Others, Student to Business keeping as the base category, has been used for the Occupation category. Except Government/PSU Employees, all other categories of employment shows a positive effect on overall mental health during lockdown.

Covid Patient: Those who tested Covid positive at least once is compared to those who have never been a Covid patient on expected overall mental health status given the other variables are held constant in the model. The ordered logit for Covid patients is greater than Non-Covid respondents, which means a Covid patient shows more effect on the overall mental when compared to the reference category.

Threat To Well Being: This is the ordered log-odds estimate of comparing the presence of a threat to wellbeing (Yes) and those facing no threat (No) to that might have a presence of a threat to well-being (MAYBE) keeping May Be as the base category. The effect of confirmed threat to well-being ordered log-odds estimate when equated to the May Be affects less than the reference category on mental health status. Whereas possibility of the No threat category displays a positive effect when compared to the reference category on overall mental health status.

Mental health status: The ordered logit for having mental health issues is less than the base category of no issues when the other variables in the model are held constant, which shows that those who are having issues has less effect on overall mental health status when related to the reference category.

Risk Analysis: Log odds of equalling the high risk and medium risk to low risk, keeping low risk as to the base category. Both high risk and medium-risk ordered log-odds displays a negative effect on overall mental health status when compared to the reference category.

The log-likelihood of the fitted model is -657.7319 at 28 degrees of freedom.

Table 1: Ordered Logit Output

\begin{tabular}{|c|c|c|}
\hline VARIABLES & Coef & t-value \\
\hline Age & & \\
\hline $18-28$ & 0.093 & 0.473 \\
\hline $29-36$ & -1.247 & 0.049 \\
\hline $39-55$ & -1.943 & 0.002 \\
\hline $56-76$ & -1.689 & 0.008 \\
\hline Gender & & \\
\hline Male & 0.284 & 0.327 \\
\hline Other & -1.093 & 0.38 \\
\hline Marital Status & & \\
\hline Married & -0.061 & 0.05 \\
\hline Unmarried & 0.862 & 0.537 \\
\hline MONTHLY INCOME & & \\
\hline 30000-50000 & -0.144 & 0.13 \\
\hline 50000-70000 & -0.777 & 0.001 \\
\hline Above 70000 & -0.462 & 0.023 \\
\hline Less than 10,000 & -0.665 & 0.008 \\
\hline OCCUPATION & & \\
\hline Daily wage workers & 0.29 & 0.73 \\
\hline Government/PSU Employees & -0.065 & 0.832 \\
\hline Housewives / Self Employed & 2.166 & 0.000 \\
\hline IT Professionals & 0.512 & 0.553 \\
\hline Others & 1.316 & 0.002 \\
\hline Student & 1.117 & 0.008 \\
\hline Covid-19 Patient & & \\
\hline Yes & 1.021 & 0.001 \\
\hline
\end{tabular}




\begin{tabular}{|c|c|c|}
\hline Threat to well-being & & \\
\hline No & -0.175 & 0.149 \\
\hline Yes & 0.518 & 0.813 \\
\hline Mental health hacks & & \\
\hline Have Issues & -0.585 & 0.005 \\
\hline Risk Analysis & & \\
\hline High & -0.846 & 0.137 \\
\hline Low & -0.696 & 0.019 \\
\hline Overall Mental Health & & \\
\hline Poor / Fair & -3.028 & 0.000 \\
\hline Fair / Good & -1.034 & 0.064 \\
\hline Good / Very Good & 0.771 & 0.081 \\
\hline Very Good/Excellent & 2.143 & 0.001 \\
\hline
\end{tabular}

CONCLUSIONS

The pandemic and lockdown have caused a lot of distress and mental health related issues. People under quarantine have expressed several difficulties in obtaining medical care and other life necessities depending on their circumstances. Upon research it was found that most affected age group in this category is 18-28 (youth). They expressed symptoms of anxiety, uncertainty and loneliness. The listed causes by them were: Severe travel restrictions, closure of all cultural centres, cancellation of sports events and public gatherings, closure of educational institutions etc. We figured out that extreme and compulsive use of social media and gaming caused higher mental illness like addiction, depression, anxiety etc.

Front line health and social service care workers were also badly affected by the pandemic. Issues like exposed environment, Occupational accidents, spread of disease, inadequate protective clothing and equipment's, low respect received, understaffed public health care system, issues related to sanitation and hygiene, extra working hours, isolation from family etc. made their both personal and work life at high risk, causing severe stress. Kids and adolescents showed higher behavioural problems and mental health related challenges. Statistics shows a high increase in child abuse and rape cases. Women and young girls polled a negative effect on their sexual health. Limited access to contraceptives and medical supplies were faced by them. Cases of increase in domestic violence and suicidal thoughts were commented by them in the survey. Informal sector workers were the hardest suffered group of people during pandemic. Loosing of jobs, less job security and poor economic conditions made their lives even harder. IT professionals including fresher's have no source of privilege in engaging with their peer groups, colleagues and lack of proper working atmosphere was a sign of mental health issues. Irrespective of impact on mental health government and PSU employees had no impact on their mental health, as they have accurate incentives, salary and job confidence. Students were using online learning platforms due to no access to schools. A higher percentage of students faced difficulties with online learning. Children from low-income family faced lack or no access to proper technological equipment's at home.

Covid-19 pandemic has affected the overall mental health of the people regardless of age and profession. The respondents have suggested different methods which can be adopted to ensure mental and physical health during these uncertain times. Practicing yoga, meditation or daily exercise can reduce stress and maintain physical health. Getting engaged by reading books, gardening, cooking, maintaining contacts with friends and family, getting enough sleep, finding new hobbies, developing one's creativity \& talents etc. can keep the mind relaxed and energetic. Getting expert's advice or consulting a psychologist /councillor can help to improve the mental health in difficult times. Most importantly keeping a good and nutritious diet is proffered.

\section{REFERENCES}

1. Saikarthik J, Saraswathi I, Siva T. Risk factors and protective factors of mental health during COVID-19 outbreak and lockdown in adult Indian population: a cross-sectional study. MedRxiv 2020;Jan 1. 
2. Khetan MS, Jaiswal KM, Vaishnao LS, Kewalramani M, Shah RJ. Effect of lockdown due to COVID-19 pandemic on mental health of pre-medical students of Maharashtra. Int J Commun Med Pub Health 2020;7(9):3524-9.

3. Schäfer SK, Sopp MR, Schanz CG, Staginnus M, Göritz AS, Michael T. Impact of COVID-19 on public mental health and the buffering effect of a sense of coherence. Psychother Psychosom 2020;89(6):386-92.

4. Das S. Mental Health and Psychosocial Aspects of COVID-19 in India: The Challenges and Responses. J Health Manage 2020;22(2):197-205.

5. Voglino G, Gualano MR, Lo Moro G, Bert F, Siliquini R. Effects of Covid-19 lockdown on mental health and sleep disturbances, results from an Italian survey. Eur J Pub Health 2020;30(Suppl 5):565-8.

6. Adams-Prassl A, Boneva T, Golin M, Rauh C. Inequality in the impact of the coronavirus shock: Evidence from real time surveys. J Pub Econom 2020;189:104245.

7. Cheng Z, Mendolia S, Paloyo AR, Savage DA, Tani M. Working parents, financial insecurity, and childcare: mental health in the time of COVID-19 in the UK. Rev Econ Household 2021;19(1):123-44.

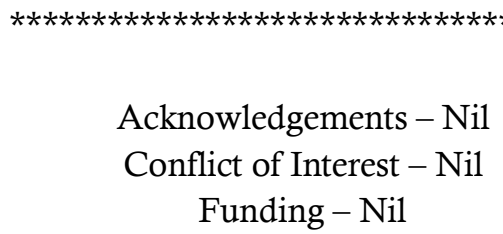

